\title{
Log Odds of Positive Lymph Node- (LODDS-) Based Competing- Risk Nomogram for Predicting Prognosis of Resected Rectal Cancer: A Development and Validation Study
}

\author{
Rui-zhe Zheng $\mathbb{D}^{1},{ }^{1}$ Jiang Xie, ${ }^{1}$ Shui-qiang Zhang, ${ }^{2}$ Wen $\mathrm{Li}^{3}{ }^{3}$ Bo Dong, ${ }^{1}$ Yin-wei Cong, \\ and Zhi-qiang Wang $\mathbb{1}^{1}$ \\ ${ }^{1}$ Department of General Surgery, Shanghai Tongren Hospital, School of Medicine, Shanghai Jiao Tong University, \\ Shanghai 200336, China \\ ${ }^{2}$ School of Engineering, Huzhou University, Huzhou 313000, China \\ ${ }^{3}$ College of Computer Science, Sichuan University, Chengdu 610065, China
}

Correspondence should be addressed to Zhi-qiang Wang; wzhqwang@qq.com

Rui-zhe Zheng and Jiang Xie contributed equally to this work.

Received 3 January 2020; Accepted 29 May 2020; Published 19 November 2020

Academic Editor: Giuseppe Nigri

Copyright ( 2020 Rui-zhe Zheng et al. This is an open access article distributed under the Creative Commons Attribution License, which permits unrestricted use, distribution, and reproduction in any medium, provided the original work is properly cited.

\begin{abstract}
Background and Aims. Cancer-specific survival (CSS) of rectal cancer (RC) is associated with several factors. We aimed to build an efficient competing-risk nomogram based on log odds of positive lymph nodes (LODDS) to predict RC survival. Methods. Medical records of 8754 patients were collected from the Surveillance, Epidemiology, and End Results (SEER) database, of 4895 patients from SEER during 2011-2014 and of 478 patients from an Eastern center as a development cohort, validation cohort, and test cohort, respectively. Univariate and multivariate competing-risk analyses were performed to build competing-risk nomogram for predicting the CSS of RC patients. Prediction efficacy was evaluated and compared with reference to the $8^{\text {th }}$ TNM classification using the factor areas under the receiver operating characteristic curve (AUC) and Brier score. Results. The competing-risk nomogram was based on 6 variables: size, $M$ stage, LODDS, $T$ stage, grade, and age. The competing-risk nomogram showed a higher AUC value in predicting the 5-year death rate due to RC than the $8^{\text {th }}$ TNM stage in the development cohort (0.81 vs. 0.76), validation cohort (0.85 vs. 0.82$)$, and test cohort (0.71 vs. 0.66). The competing-risk nomogram also showed a higher Brier score in predicting the 5 -year death rate due to RC than the $8^{\text {th }}$ TNM stage in the development cohort $(0.120$ vs. 0.127$)$, validation cohort $(0.123$ vs. 0.128$)$, and test cohort $(0.202$ vs. 0.226$)$. Conclusion. We developed and validated a competing-risk nomogram for RC death, which could provide the probability of survival averting competing risk to facilitate clinical decision-making.
\end{abstract}

\section{Introduction}

Rectal cancer (RC) is one of the most commonly diagnosed cancers worldwide [1]. Past studies have suggested that the prognosis of RC may be influenced by several factors [2-4], such as gender, age, carcinoembryonic antigen (CEA), location, pT, pN, tumor size, lymph node (LN) metastasis, concomitant diseases, surgical procedure, residual cancer, and complications. The involvement of a vari- ety of factors has added to the complexity of the predicting model. The survival of RC may also be contended by several risks. The competing-risk nomogram model reduces the risk of influencing factors, which gives it a good predicting accuracy [5].

Currently, the American Joint Committee on Cancer (AJCC) $8^{\text {th }} \mathrm{N}$ staging is the most widely used staging platform for predicting RC survival [6]. However, accurate N staging relies on sufficient histological examination of at 
TABLE 1: Clinical characteristics of patients with rectal cancer in different cohorts.

\begin{tabular}{|c|c|c|c|c|}
\hline & $\begin{array}{l}\text { Development cohort } \\
\qquad N=8754\end{array}$ & $\begin{array}{c}\text { Validation cohort } \\
\qquad N=4895\end{array}$ & $\begin{array}{l}\text { Test cohort } \\
N=478\end{array}$ & $P$ \\
\hline $\operatorname{Sex}$ & & & & $<0.001$ \\
\hline Female & $4202(48.00 \%)$ & $2220(45.35 \%)$ & $183(38.28 \%)$ & \\
\hline Male & $4552(52.00 \%)$ & $2675(54.65 \%)$ & $295(61.72 \%)$ & \\
\hline Grade & & & & $<0.001$ \\
\hline $\mathrm{I} / \mathrm{II}$ & $7581(86.60 \%)$ & $4214(86.09 \%)$ & $373(78.03 \%)$ & \\
\hline III/IV & $1173(13.40 \%)$ & $681(13.91 \%)$ & $105(21.97 \%)$ & \\
\hline Age (year) & & & & $<0.001$ \\
\hline$\leq 49$ & $1074(12.27 \%)$ & $738(15.08 \%)$ & $43(9.00 \%)$ & \\
\hline $50-59$ & $1935(22.10 \%)$ & $1242(25.37 \%)$ & $127(26.57 \%)$ & \\
\hline $60-69$ & $2015(23.02 \%)$ & $1219(24.90 \%)$ & $116(24.27 \%)$ & \\
\hline$\geq 70$ & $3730(42.61 \%)$ & $1696(34.65 \%)$ & $192(40.17 \%)$ & \\
\hline Size $(\mathrm{cm})$ & & & & $<0.001$ \\
\hline$\leq 2.0$ & $1546(17.66 \%)$ & $806(16.47 \%)$ & $15(3.14 \%)$ & \\
\hline$\leq 4.0$ & $3593(41.04 \%)$ & $1855(37.90 \%)$ & $142(29.71 \%)$ & \\
\hline$>4.0$ & $3615(41.30 \%)$ & $2234(45.64 \%)$ & $321(67.15 \%)$ & \\
\hline T stage & & & & $<0.001$ \\
\hline $\mathrm{T} 1$ & $1503(17.17 \%)$ & $830(16.96 \%)$ & $10(2.09 \%)$ & \\
\hline $\mathrm{T} 2$ & $2632(30.07 \%)$ & $1158(23.66 \%)$ & $75(15.69 \%)$ & \\
\hline T3 & $4141(47.30 \%)$ & $2425(49.54 \%)$ & 368 (76.99\%) & \\
\hline $\mathrm{T} 4 \mathrm{a}$ & $271(3.10 \%)$ & $306(6.25 \%)$ & $12(2.51 \%)$ & \\
\hline $\mathrm{T} 4 \mathrm{~b}$ & $207(2.36 \%)$ & $176(3.60 \%)$ & $13(2.72 \%)$ & \\
\hline$M$ stage & & & & $<0.001$ \\
\hline M0 & 8707 (99.46\%) & $4265(87.13 \%)$ & 467 (97.70\%) & \\
\hline M1a & $31(0.35 \%)$ & $430(8.78 \%)$ & $7(1.46 \%)$ & \\
\hline M1b & $16(0.18 \%)$ & $200(4.09 \%)$ & $4(0.84 \%)$ & \\
\hline pN (median, IQR) & $0(0,1)$ & $0(0,2)$ & $0(0,2)$ & $<0.001$ \\
\hline $\mathrm{nN}$ (median, IQR) & $12(8,18)$ & $15(11,20)$ & $4(2,6)$ & $<0.001$ \\
\hline LODDS (median, IQR) & $-2.94(-3.50,-1.78)$ & $-3.14(-3.56,-1.72)$ & $-1.61(-2.40,-0.59)$ & $<0.001$ \\
\hline N stage & & & & $<0.001$ \\
\hline No & $5858(66.92 \%)$ & $2984(60.96 \%)$ & $276(57.74 \%)$ & \\
\hline N1a & $926(10.58 \%)$ & $537(10.97 \%)$ & $70(14.64 \%)$ & \\
\hline N1b & $891(10.18 \%)$ & $542(11.07 \%)$ & $61(12.76 \%)$ & \\
\hline $\mathrm{N} 2 \mathrm{a}$ & $591(6.75 \%)$ & $415(8.48 \%)$ & $4(0.84 \%)$ & \\
\hline $\mathrm{N} 2 \mathrm{~b}$ & $488(5.57 \%)$ & $417(8.52 \%)$ & $45(9.41 \%)$ & \\
\hline
\end{tabular}

pN: positive lymph nodes; $\mathrm{nN}$ : negative lymph nodes; LODDS: log odds of positive lymph nodes. IQR: interquartile range.

least 12 LNs. A lesser number of examined LNs could lead to staging migration and the mistake of indulging in further treatment. Log odds of positive lymph nodes (LODDS) represent the logarithm of retrieving the $\mathrm{LN}$ ratio between the probability of positive and negative LNs, which signifies greater predicting ability relative to that by the conventional LN staging in gastrointestinal cancers [7-9].

In this study, we developed and validated a LODDSbased competing-risk nomogram using a large populationbased cohort and tested the nomogram in another independent cohort.

\section{Methods}

2.1. Study Design and Data Collection. We performed this study based on the incidence and survival data obtained from the Surveillance, Epidemiology, and End Results (SEER 18) database and the Shanghai Tongren Hospital. We collected medical records of resected RC patients of age $>18$ years with a known number of histologically examined LN and histologically positive LN. Patients with other tumors or those without histological confirmation were excluded from the study. We also excluded patients with missing data for development and validation of the model, inclusive of unknown gender, 
TABLE 2: Competing-risk survival analysis for exploring potential risk factors for prognosis of resected rectal cancer.

\begin{tabular}{|c|c|c|c|c|c|}
\hline \multirow{2}{*}{ Characteristics } & \multicolumn{2}{|c|}{ Univariate model } & \multicolumn{3}{|c|}{ Final multivariate model } \\
\hline & SHR (95\% CI) & $P$ & Beta & SHR (95\% CI) & $P$ \\
\hline \multicolumn{6}{|l|}{ Age (year) } \\
\hline$\leq 49$ & Reference & & & Reference & \\
\hline $50-59$ & $1.12(0.93-1.34)$ & 0.240 & 0.276 & $1.32(1.10-1.58)$ & 0.003 \\
\hline $60-69$ & $1.33(1.11-1.58)$ & 0.002 & 0.435 & $1.54(1.29-1.85)$ & $<0.001$ \\
\hline$\geq 70$ & $2.10(1.79-2.47)$ & $<0.001$ & 0.863 & $2.37(2.02-2.79)$ & $<0.001$ \\
\hline \multicolumn{6}{|l|}{ T stage } \\
\hline $\mathrm{T} 1$ & Reference & & & Reference & \\
\hline $\mathrm{T} 2$ & $1.61(1.32-1.97)$ & $<0.001$ & 0.293 & $1.34(1.08-1.66)$ & 0.007 \\
\hline $\mathrm{T} 3$ & $4.24(3.53-5.08)$ & $<0.001$ & 0.935 & $2.55(2.07-3.13)$ & $<0.001$ \\
\hline $\mathrm{T} 4 \mathrm{a}$ & $6.83(5.30-8.81)$ & $<0.001$ & 1.232 & $3.43(2.57-4.58)$ & $<0.001$ \\
\hline $\mathrm{T} 4 \mathrm{~b}$ & $10.32(7.95-13.39)$ & $<0.001$ & 1.761 & $5.82(4.34-7.79)$ & $<0.001$ \\
\hline \multicolumn{6}{|l|}{ M stage } \\
\hline M0 & Reference & & & Reference & \\
\hline M1a & $5.13(3.64-7.23)$ & $<0.001$ & 0.978 & $2.66(1.84-3.85)$ & $<0.001$ \\
\hline M1b & $7.61(5.34-10.85)$ & $<0.001$ & 1.297 & $3.66(2.47-5.43)$ & $<0.001$ \\
\hline LODDS & $1.51(1.47-1.55)$ & $<0.001$ & & & \\
\hline \multicolumn{6}{|l|}{ Grade } \\
\hline $\mathrm{I} / \mathrm{II}$ & Reference & & & Reference & \\
\hline III/IV & $1.87(1.68-2.09)$ & $<0.001$ & 0.249 & $1.87(1.68-2.09)$ & $<0.001$ \\
\hline \multicolumn{6}{|l|}{ Size $(\mathrm{cm})$} \\
\hline$\leq 2.0$ & Reference & & & Reference & \\
\hline$\leq 4.0$ & $2.05(1.74-2.41)$ & $<0.001$ & 0.267 & $1.31(1.09-1.56)$ & $<0.001$ \\
\hline$>4.0$ & $2.82(2.41-3.31)$ & $<0.001$ & 0.367 & $1.46(1.21-1.75)$ & $<0.001$ \\
\hline \multicolumn{6}{|l|}{ Sex } \\
\hline Female & Reference & & & & \\
\hline Male & $1.01(0.92-1.10)$ & 0.910 & & & \\
\hline
\end{tabular}

SHR: subdistribution hazard ratio; LODDS: log odds of positive lymph nodes.

age, grade, $\mathrm{T}$ stage, $\mathrm{M}$ stage, $\mathrm{N}$ stage, tumor size, location, examined LN numbers, positive LN numbers, and followup of $<3$ months.

Patients whose data were collected from the SEER database during 2004-2010 were included in the development cohort, while those whose data were collected during 20112014 were included in the validation cohort. Patients diagnosed with RC during 2008-2013 were retrieved as the test cohort. We collected patients' clinicopathological variables including age, gender, tumor location, TNM staging, grade of tumor, histological grade, number of examined LNs, number of positive LNs, tumor size, and survival months. LODDS was defined as loge $[(\mathrm{pN}+0.5) /(\mathrm{nN}+0.5)]$, where $\mathrm{pN}$ is the number of positive lymph nodes and $\mathrm{nN}$ is the number of negative nodes retrieved [10].

2.2. Statistical Analysis. In the competitive-risk analysis, we defined RC-specific death as the primary outcome and other causes of death as competing events. Univariate and multivariate proportional subdistribution hazard models were applied to calculate the adjusted subdistribution hazard ratio (SHR) for each included variable. Variables with $P<0.1$ in the univariate analysis and $P<0.05$ in the initial multivariate analysis were finally included to develop the competing-risk nomogram. We presented the model visible as a nomogram based on Fine and Gray's model [11].

This model was internally developed, externally validated, and then tested in 3 independent cohorts. A calibration plot was employed to compare the predicted to observed probabilities of death at a certain point of time [12]. Pairs of predicted and observed probabilities lay on a 45-degree angle line if both the probabilities matched well and proved the model ideal. We then compared this model to the $8^{\text {th }}$ TNM staging by several assessment methods. The area under the curve (AUC) and higher Brier score were used to assess discrimination of the competing-risk nomogram [13-15]. All statistical analyses were performed by $\mathrm{R}$ version 3.3.3 software (Institute for Statistics and Mathematics, Vienna, Austria; http://www.r-project.org).

\section{Results}

3.1. Patients. The final trial sample included 8754 patients in the development cohort, 4895 in the validation cohort, and 478 in the test cohort (baseline details given in Table 1). Higher age ( $\leq 49$ years, $50-59$ years, $60-69$ years, $\geq 70$ years, 


\begin{tabular}{|c|c|c|c|c|c|c|c|c|c|c|c|}
\hline \multirow{3}{*}{ M stage } & Points & 10 & 20 & 30 & 40 & 50 & 60 & 70 & 80 & 90 & 100 \\
\hline & & & & & & & M1a & & & & \\
\hline & & & & M0 & & & & $11 \mathrm{~b}$ & & & \\
\hline
\end{tabular}

LODDS

\begin{tabular}{llllllllllll}
\hline-6 & -5 & -4 & -3 & -2 & -1 & 0 & 1 & 2 & 3 & 4 & 5
\end{tabular}

T stage

\begin{tabular}{cccc}
\multicolumn{2}{c}{ T2 } & T4a \\
T1 & T3 & T4b
\end{tabular}

Grade

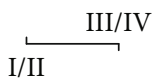

Size $(\mathrm{cm})$

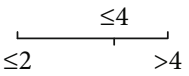

Age (year)

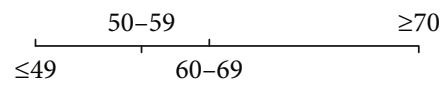

Total-points-to-outcome nomogram:

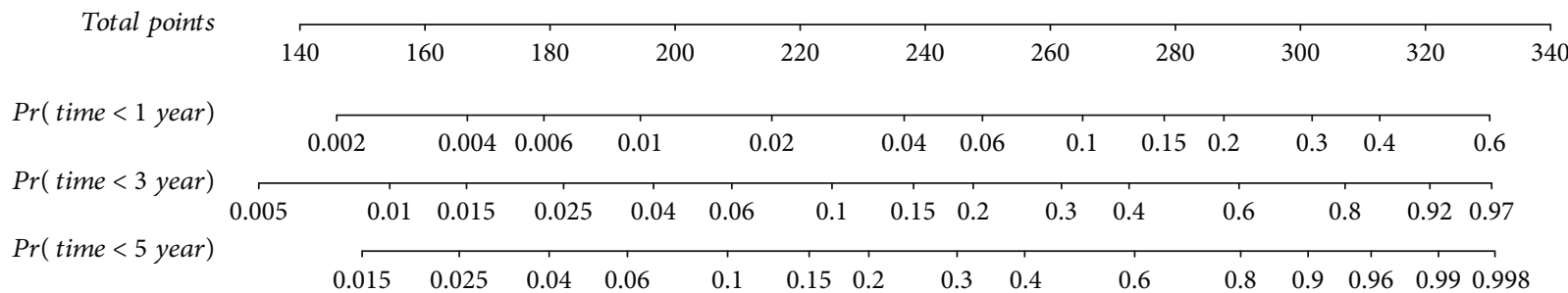

FIGURE 1: Nomogram predicted 1-, 3-, and 5-year cancer-specific death for patients with resected rectal cancer based on 6 available clinical characteristics. For each predictor, read the points assigned on the $0-100$ scale at the top and then add these points. Find the number on the "total points" scale and then read the corresponding predictions of 1-, 3-, and 5-year cancer-specific death risk using the competing-risk model.

SHR 1.32, 1.54, 2.37, $P<0.05)$, higher T stage $(\mathrm{T} 1-4 \mathrm{~b}, \mathrm{SHR}$ $1.34,2.55,3.43,5.82, P=0.007$, respectively, $P<0.001)$, higher M stage (M0-M1b, SHR 2.66, 3.66, respectively, $P<$ 0.001 ), high LODDS (SHR 1.51, $P<0.001$ ), high grade (SHR 1.87, $P<0.001)$, and larger size $(\leq 2 \mathrm{~cm},>2, \leq 4 \mathrm{~cm}$, $>4 \mathrm{~cm}$, SHR 1.31, 1.46, respectively, $P<0.001)$ were associated with a poor prognosis (Table 2). The above-mentioned risk factors were considered in the final development of the competing-risk nomogram.

The RC cause-specific death predicting model of nomogram was established based on selected prognostic factors (Figure 1). The nomogram revealed that LODDS was the most important risk factor for RC prognosis, followed by $\mathrm{T}$ stage, $M$ stage, age, size, and grade. Each subtype of the variables was assigned a specific score. A straight line to determine the estimated probability of survival was drawn at each time point on the total point scale, according to the total point.

3.2. Evaluation of the Competing-Risk Nomogram. The calibration curves given in (Figure 2) show the dots close to a $45^{\circ}$ diagonal line. The figure also suggests that the competing-risk nomogram showed good calibration for predicting 1-year, 3-year, and 5-year RC death in the development, validation, and test cohorts, respectively.

We used both AUC and Brier score to evaluate the diagnosis value and accuracy of the nomogram model (Table 3 ). The AUC value was greater in the nomogram model than in the conventional TNM staging method for the development cohort (1-year: 0.82 vs. $0.74,2$-year: 0.84 vs. $0.77,3$-year: 0.83 vs. $0.77,4$-year: 0.82 vs. 0.77 , and 5 -year: 0.81 vs. 0.76 , respectively, $P<0.05$; Figure 3 ); validation cohort (1-year: 0.84 vs. $0.79,2$-year: 0.87 vs. $0.81,3$-year: 0.87 vs. 0.82 , 4 -year: 0.86 vs. 0.82 , and 5 -year: 0.85 vs. 0.82 , respectively, $P<0.05$; Figure 3 ), and test cohort (1-year: 0.75 vs. $0.69,2$-year: 0.72 vs. 0.69 , 3 -year: 0.73 vs. 0.68 , 4 -year: 0.71 vs. 0.67 , and 5 -year: 0.71 vs. 0.66 , respectively, $P<0.05$; Figure 3 ).

The accuracy of the Brier score was better with the nomogram than with the conventional TNM staging approach in the development cohort (1-year: 0.04 vs. 0.042, 2-year: 0.067 vs. 0.073 , 3-year: 0.090 vs. $0.097,4$-year: 0.108 vs. 0.116 , and 5-year: 0.120 vs. 0.127 , respectively, $P<0.05$; 


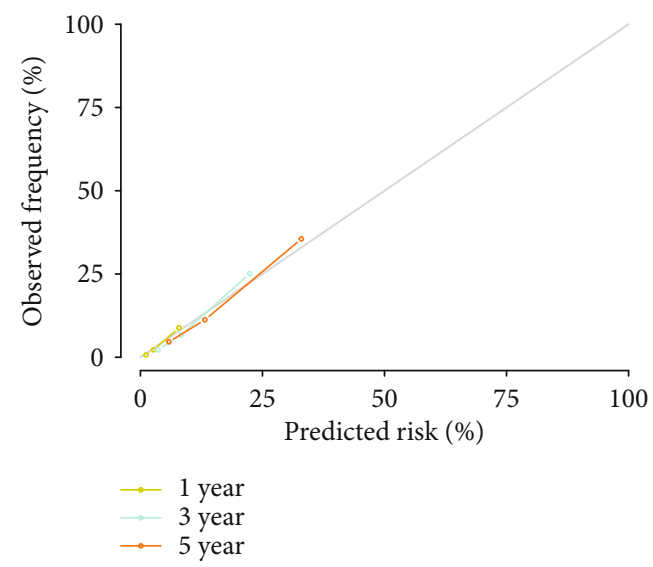

(a)

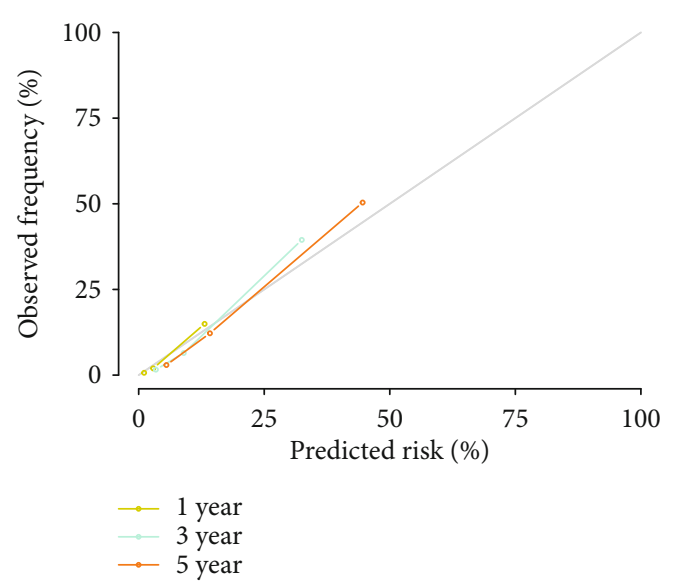

(b)

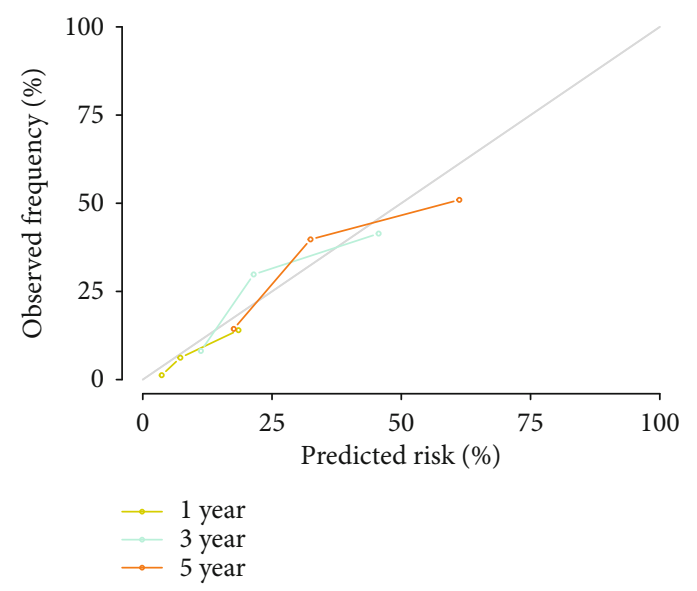

(c)

Figure 2: The calibration curves for predicting patient survival at 1-, 3-, and 5-year points in the development cohort (a), validation cohort (b), and test cohort (c). Nomogram-predicted cancer-specific survival is plotted on the $x$-axis; actual cancer-specific survival is plotted on the $y$ -axis. A plot along the 45-degree line indicates a perfect calibration model, wherein the predicted probabilities are identical to the actual outcomes.

TABLE 3: Comparison of AUC and Brier score between the nomogram and $8^{\text {th }}$ TNM stage in patients with rectal cancer.

\begin{tabular}{|c|c|c|c|c|c|c|}
\hline \multirow[b]{2}{*}{ Time } & \multicolumn{2}{|c|}{ Development cohort } & \multicolumn{2}{|c|}{ Validation cohort } & \multicolumn{2}{|c|}{ Test cohort } \\
\hline & Nomogram & $8^{\text {th }}$ TNM stage & Nomogram & $8^{\text {th }}$ TNM stage & Nomogram & $8^{\text {th }}$ TNM stage \\
\hline \multicolumn{7}{|l|}{$A U C$} \\
\hline 1 year & 0.82 & 0.74 & 0.84 & 0.79 & 0.75 & 0.69 \\
\hline 2 years & 0.84 & 0.77 & 0.87 & 0.81 & 0.72 & 0.69 \\
\hline 3 years & 0.83 & 0.77 & 0.87 & 0.82 & 0.73 & 0.68 \\
\hline 4 years & 0.82 & 0.77 & 0.86 & 0.82 & 0.71 & 0.67 \\
\hline 5 years & 0.81 & 0.76 & 0.85 & 0.82 & 0.71 & 0.66 \\
\hline \multicolumn{7}{|c|}{ Brier score } \\
\hline 1 years & 0.040 & 0.042 & 0.047 & 0.051 & 0.064 & 0.065 \\
\hline 2 years & 0.067 & 0.073 & 0.077 & 0.084 & 0.134 & 0.147 \\
\hline 3 years & 0.090 & 0.097 & 0.097 & 0.104 & 0.170 & 0.192 \\
\hline 4 years & 0.108 & 0.116 & 0.113 & 0.119 & 0.188 & 0.205 \\
\hline 5 years & 0.120 & 0.127 & 0.123 & 0.128 & 0.202 & 0.226 \\
\hline
\end{tabular}

AUC: area under the receiver operating characteristic curve. 


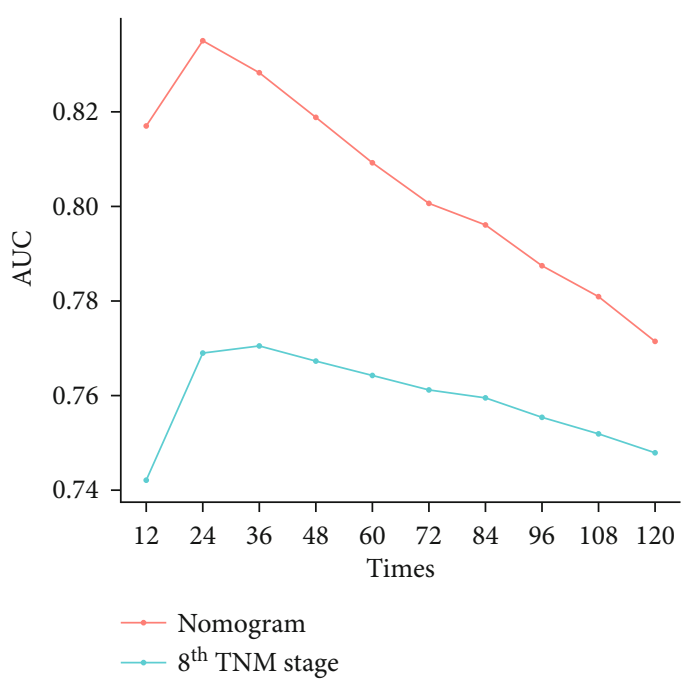

(a)

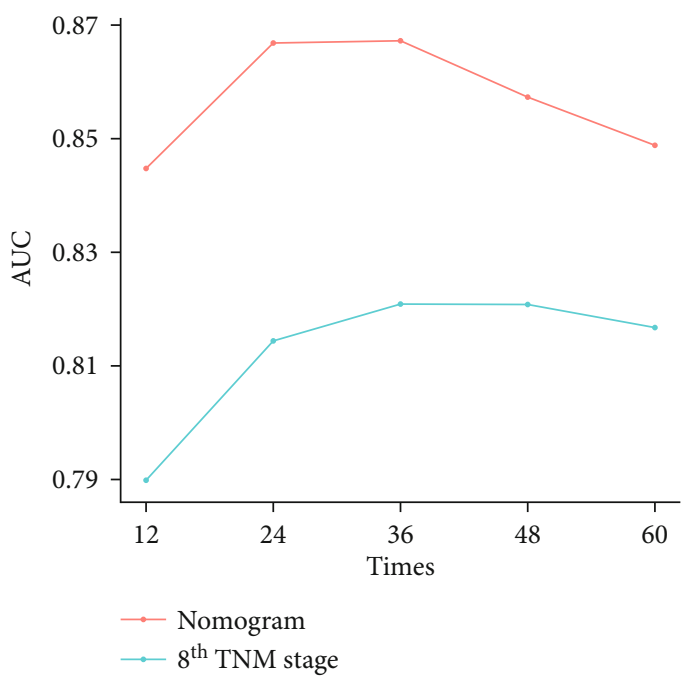

(c)

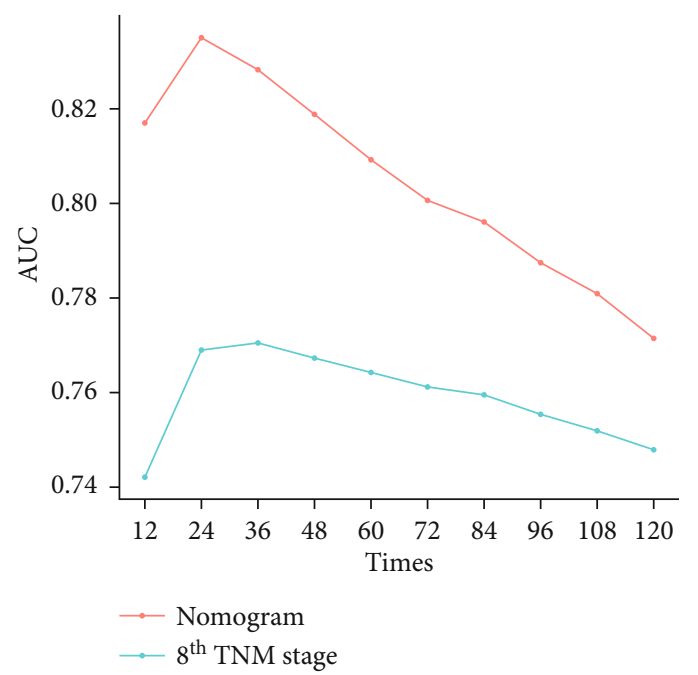

(b)

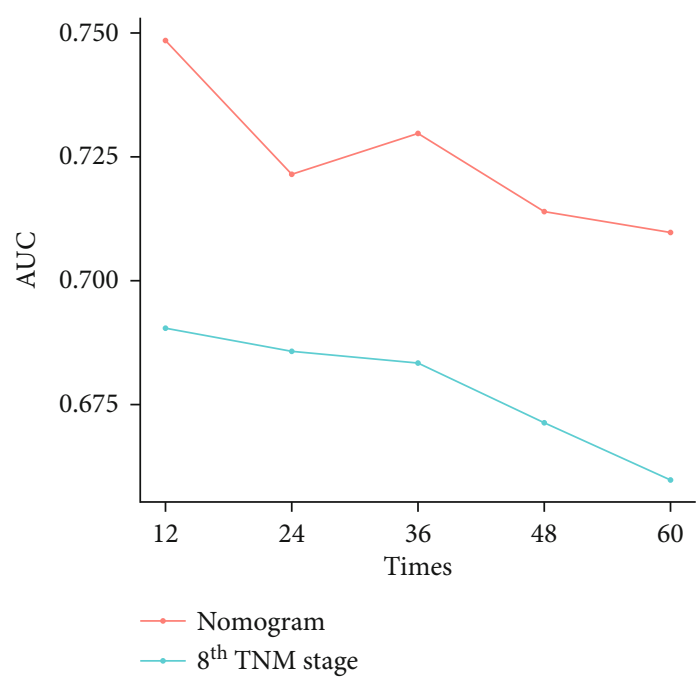

(d)

FIGURE 3: AUC of the nomogram and $8^{\text {th }}$ TNM staging in the prediction of prognosis of patients from the 1-year to the 10-year point in the development cohort (a), development cohort with bootstrapped validation (b), validation cohort (c), and test cohort (d). AUC: areas under the receiver operating characteristic curve.

Figure 4), validation cohort (1-year: 0.047 vs. 0.051, 2-year: 0.077 vs. $0.084,3$-year: 0.097 vs. 0.104 , 4 -year: 0.113 vs. 0.119 , and 5-year: 0.123 vs. 0.128 , respectively, $P<0.05$; Figure 4), and test cohort (1-year: 0.064 vs. 0.065, 2-year: 0.134 vs. $0.147,3$-year: 0.170 vs. 0.192 , 4 -year: 0.188 vs. 0.205 , and 5 -year: 0.202 vs. 0.226 , respectively, $P<0.05$; Figure 4).

\section{Discussion}

As one of the most common cancer, RC has received much attention in both the Western and Eastern countries $[1,16]$. The prediction of prognosis is critically important for accurate clinical decision-making. To the best of our knowledge, this is the first study to develop a competing-risk nomogram for predicting RC death. In this study, we developed and validated a new competing-risk nomogram model based on a large Western cohort from SEER, which we then tested on an Eastern cohort. We found that this model demonstrated greater clinical predicting ability than the $8^{\text {th }}$ TNM staging approach performed conventionally in RC patients.

The new $8^{\text {th }}$ AJCC TNM staging stresses the personalization of the predicting classification [6]. Thus, the more effective factors of cancer are paid more attention [2, 4]. In a prior study, gender, age, CEA, location, $\mathrm{pT}, \mathrm{pN}$, size, LN metastasis, concomitant diseases, surgical procedure, residual cancer, and complications were considered factors that could affect the prognosis of $\mathrm{RC}[2,4,17]$. In fact, several factors are possibly associated with many other death causes, such as other cancers, concomitant diseases, complications, and accidents. As these causes compete with the RC cause-specific death, it adds bias to prognosis prediction. For accurately predicting the RC cause-specific deaths, we applied the competing-risk nomogram to reduce the potential bias [11]. The nomogram showed that LODDS was the most impactful contributing factor for prognosis, followed by $\mathrm{T}$ stage, $\mathrm{M}$ stage, age, size, 


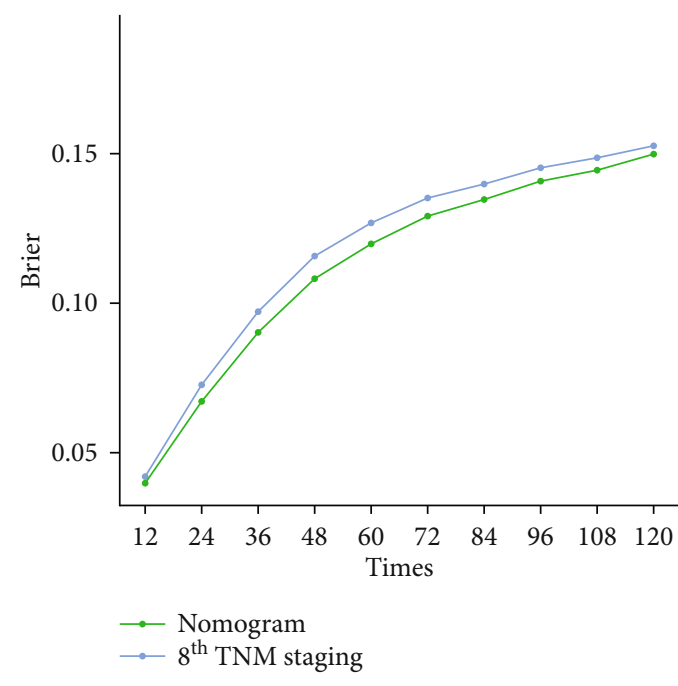

(a)

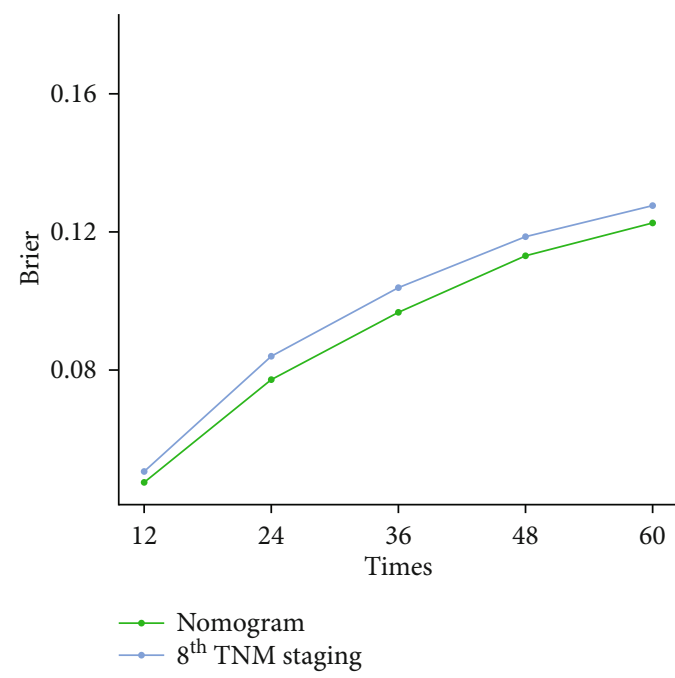

(c)

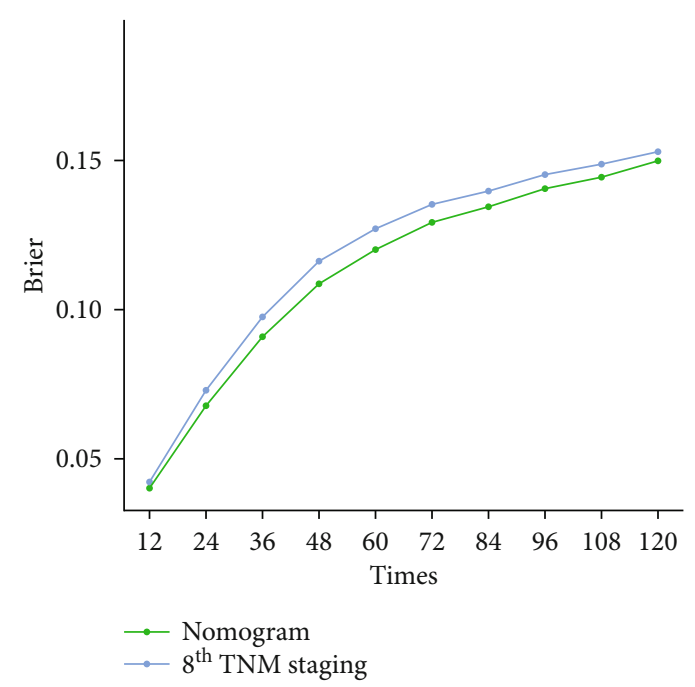

(b)

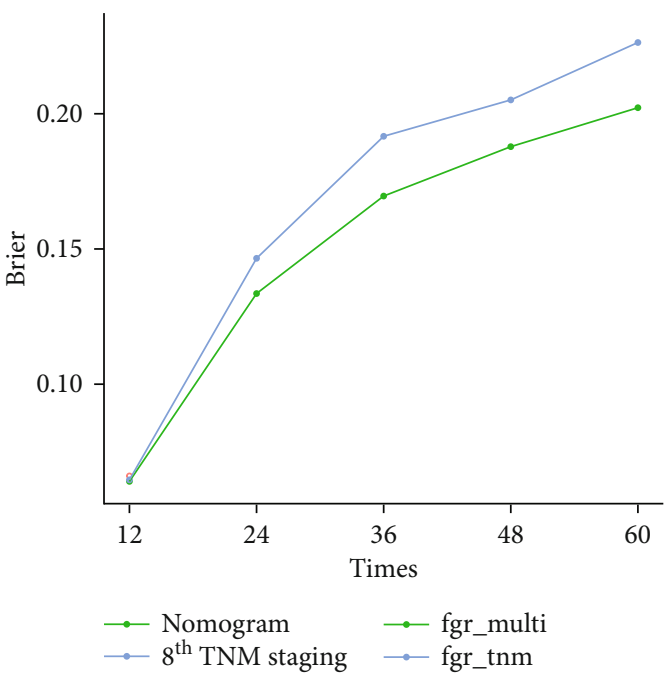

(d)

FIGURE 4: Brier score of the nomogram and $8^{\text {th }}$ TNM staging in the prediction of prognosis of patients from the 1-year to the 10-year point in the development cohort (a), development cohort with bootstrapped validation (b), validation cohort (c), and test cohort (d).

and grade of the tumor. Our proposed model demonstrated superior prediction ability as compared to the $8^{\text {th }}$ AJCC TNM staging in terms of higher AUC and lower Brier score. Moreover, in the past, certain models had been developed and validated in the Western cohorts, which did not show clinical applicability in Eastern cohorts. In this study, we also tested this model in an Eastern cohort, which increased the scope of application.

LN is an important prognostic factor for overall survival. The AJCC $\mathrm{N}$ classification is widely used in clinical practice [18], although its accuracy remains debatable in gastrointestinal cancers $[7,8,19]$. This staging approach is based on the absolute number of positive LNs, which in turn relies on sufficient numbers of examined LNs to fully assess the metastatic status. AJCC recommends at least $12 \mathrm{LNs}$, while some study recommends even more [20-22]. This staging approach involves complex functionalities for surgeons and pathologists and can hence result in migration. On the other hand, LODDS is a logarithm of the probability between being a positive or negative LN when it is retrieved [7]. It has been applied in gastrointestinal cancers to improve the accuracy of LN staging for predicting the prognosis [7-10]. In this study, we collected the LODDS of each patient and performed univariate and multivariate analyses to select factors for the development of a competing-risk nomogram. The resultant nomogram indicated that LODDS is the most impactful contributing factor of prognosis.

We identified some limitations in this study. First, this is a retrospective trial based on the SEER database and an Eastern cohort data, which may have caused selection bias. The missing data could also influence the analysis of prognosis and the development of the model. The large number of this trial may reduce this bias. Second, AJCC pays attention to the new therapy and biomarkers, which could influence the 
prognosis of RC. However, as the current database did not contain this information, we expect more innovations and trials of this field in the future.

\section{Conclusion}

In conclusion, we developed a new competing-risk nomogram based on LODDS to predict the prognosis of RC. This model reduced the bias of competing with other death causes and staging migration of the conventional $\mathrm{N}$ staging. The new staging system shows greater prediction accuracy and better ability than the conventional $8^{\text {th }}$ TNM staging approach for both the Eastern and Western cohorts.

\section{Data Availability}

The raw data used to support the findings of this study are included within the article.

\section{Ethical Approval}

This study was approved by the Ethics Committee of the Shanghai Tongren Hospital Affiliated to Shanghai Jiao Tong University School of Medicine.

\section{Conflicts of Interest}

The authors declare that they have no conflicts of interest.

\section{Authors' Contributions}

All authors have read and approved the final manuscript. We certify that all authors have made substantial contributions so as to qualify for authorship. Rui-zhe Zheng and Jiang Xie contributed equally to this work.

\section{Acknowledgments}

This study was supported by the Fund of Science Committee of Changning District, Shanghai, China (Nos. YXMZK004 and MYGZS005).

\section{References}

[1] R. L. Siegel, K. D. Miller, and A. Jemal, "Cancer statistics, 2018," CA: A Cancer Journal for Clinicians, vol. 68, no. 1, pp. 7-30, 2018.

[2] J. Peng, Y. Ding, S. Tu et al., "Prognostic nomograms for predicting survival and distant metastases in locally advanced rectal cancers," PLoS ONE, vol. 9, no. 8, p. e106344, 2014.

[3] V. Valentini, R. G. P. M. van Stiphout, G. Lammering et al., "Nomograms for predicting local recurrence, distant metastases, and overall survival for patients with locally advanced rectal cancer on the basis of European randomized clinical trials," Journal of Clinical Oncology, vol. 29, no. 23, pp. 3163-3172, 2011.

[4] W. van Gijn, R. G. P. M. van Stiphout, C. J. H. van de Velde et al., "Nomograms to predict survival and the risk for developing local or distant recurrence in patients with rectal cancer treated with optional short-term radiotherapy," Annals of Oncology, vol. 26, no. 5, pp. 928-935, 2015.
[5] S. Kang, B. H. Nam, J. Y. Park et al., "Risk assessment tool for distant recurrence after platinum-based concurrent chemoradiation in patients with locally advanced cervical cancer: a Korean gynecologic oncology group study," Journal of Clinical Oncology, vol. 30, no. 19, pp. 2369-2374, 2012.

[6] M. B. Amin, F. L. Greene, S. B. Edge et al., "The Eighth Edition AJCC Cancer Staging Manual: Continuing to build a bridge from a population-based to a more "personalized" approach to cancer staging," CA: A Cancer Journal for Clinicians, vol. 67, no. 2, pp. 93-99, 2017.

[7] J.-P. Pei, C.-D. Zhang, Y.-C. Fan, and D.-Q. Dai, "Comparison of different lymph node staging systems in patients with resectable colorectal cancer," Frontiers in Oncology, vol. 8, 2019.

[8] Y.-Y. Zhou, X.-J. Du, C.-H. Zhang et al., “Comparison of three lymph node staging schemes for predicting the outcome in patients with small bowel adenocarcinoma: a populationbased cohort and international multicentre cohort study," EBioMedicine, vol. 41, pp. 276-285, 2019.

[9] G. Spolverato, A. Ejaz, Y. Kim et al., "Prognostic performance of different lymph node staging systems after curative intent resection for gastric adenocarcinoma," Annals of Surgery, vol. 262, no. 6, pp. 991-998, 2015.

[10] D. D. Smith, R. A. Nelson, and R. E. Schwarz, "A comparison of five competing lymph node staging schemes in a cohort of resectable gastric cancer patients," Annals of Surgical Oncology, vol. 21, no. 3, pp. 875-882, 2014.

[11] J. P. Fine and R. J. Gray, "A proportional hazards model for the subdistribution of a competing risk," Journal of the American Statistical Association, vol. 94, no. 446, pp. 496-509, 1999.

[12] J. S. Hyams, S. Davis Thomas, N. Gotman et al., "Clinical and biological predictors of response to standardised paediatric colitis therapy (PROTECT): a multicentre inception cohort study," The Lancet, vol. 393, no. 10182, pp. 1708-1720, 2019.

[13] J. A. Hanley and B. J. McNeil, "A method of comparing the areas under receiver operating characteristic curves derived from the same cases.," Radiology, vol. 148, no. 3, pp. 839843,1983

[14] G. Gandaglia, N. Fossati, E. Zaffuto et al., "Development and internal validation of a novel model to identify the candidates for extended pelvic lymph node dissection in prostate cancer," European Urology, vol. 72, no. 4, pp. 632-640, 2017.

[15] D. Callegaro, R. Miceli, S. Bonvalot et al., "Development and external validation of two nomograms to predict overall survival and occurrence of distant metastases in adults after surgical resection of localised soft-tissue sarcomas of the extremities: a retrospective analysis," The Lancet Oncology, vol. 17, no. 5, pp. 671-680, 2016.

[16] R. L. Siegel, K. D. Miller, S. A. Fedewa et al., "Colorectal cancer statistics, 2017," CA: A Cancer Journal for Clinicians, vol. 67, no. 3, pp. 177-193, 2017.

[17] K. Kawai, E. Sunami, H. Yamaguchi et al., "Nomograms for colorectal cancer: a systematic review," World Journal of Gastroenterology, vol. 21, no. 41, pp. 11877-11886, 2015.

[18] B. Zhao, R. A. Gabriel, F. Vaida, N. E. Lopez, S. Eisenstein, and B. M. Clary, "Predicting Overall Survival in Patients with Metastatic Rectal Cancer: a Machine Learning Approach," Journal of gastrointestinal surgery: official journal of the Society for Surgery of the Alimentary Tract, 2019.

[19] Z.-X. Wang, M.-Z. Qiu, Y.-M. Jiang, Z.-W. Zhou, G.-X. Li, and R.-H. Xu, "Comparison of prognostic nomograms based on 
different nodal staging systems in patients with resected gastric cancer," Journal of Cancer, vol. 8, no. 6, pp. 950-958, 2017.

[20] S. Wu, J.-N. Chen, Q.-W. Zhang et al., "A new metastatic lymph node classification-based survival predicting model in patients with small bowel adenocarcinoma: a derivation and validation study," EBioMedicine, vol. 32, pp. 134-141, 2018.

[21] J. Kehoe and V. P. Khatri, "Staging and prognosis of colon cancer.," Surgical Oncology Clinics of North America, vol. 15, no. 1, pp. 129-146, 2006.

[22] S. L. Wong, H. Ji, B. K. Hollenbeck, A. M. Morris, O. Baser, and J. D. Birkmeyer, "Hospital lymph node examination rates and survival after resection for colon cancer," JAMA, vol. 298, no. 18, pp. 2149-2154, 2007. 